\title{
Decayed, Missing, and Filled Teeth Index
}

National Cancer Institute

\section{Source}

National Cancer Institute. Decayed, Missing, and Filled Teeth Index. NCI Thesaurus. Code C94566.

A system used to quantitate and illustrate the current state of a person's dentition. It is a sum of the total number of teeth that are decayed, missing, or filled. It is calculated either for 32 permanent teeth, or for 28 teeth (excluding the third molars). 